\title{
Lisina digestível para leitoas em fase de crescimento
}

\author{
Digestible lysine for growing gilts
}

\section{Gabriel Cipriano Rocha ${ }^{I^{*}}$ Juarez Lopes Donzele ${ }^{\mathrm{II}}$ Rita Flavia Miranda de Oliveira Donzele ${ }^{\mathrm{II}}$ Francisco Carlos de Oliveira da Silva ${ }^{\mathrm{II}}$ Charles Kiefer $^{\mathrm{IV}}$ Leandro Alebrante $^{\mathrm{I}}$ Pedro Veiga Rodrigues PaulinoI Nicola Vergara Lopes Serãov}

\section{RESUMO}

Oitenta leitoas $(24,2 \pm 1,52 \mathrm{~kg})$ com alto potencial genético para deposição de carne na carcaça foram distribuídas em experimento de blocos ao acaso para avaliar cinco níveis de lisina digestivel $(\mathrm{Ld})\left(9,10,11,12\right.$ e $\left.13 \mathrm{~g} \mathrm{~kg}^{-1}\right)$ durante a fase de crescimento (63 a 103 dias de idade). Os animais foram alojados em pares e alimentados à vontade. No início e ao final do periodo experimental, as leitoas foram pesadas e submetidas à análise de ultrassom para avaliação da área de olho de lombo (AOL) e espessura de toucinho (ET). Os níveis de Ld proporcionaram aumento linear $(P<0,05)$ do ganho de peso diário $(G P D) e$, apesar dessa variação, não houve aumento no valor absoluto do GPD a partir do nivel de $12 \mathrm{~g} \mathrm{~kg}^{-1}$ de Ld. A conversão alimentar $(C A)$ reduziu $(P<0,01)$ de forma quadrática até o nível estimado de $11,9 \mathrm{~g} \mathrm{~kg}^{-1}$ de Ld. A AOL das leitoas aumentou $(P<0,05)$ de forma linear. No entanto, o modelo "Linear Response Plateau" foi o que melhor se ajustou aos dados, estimando em 12,5g $\mathrm{kg}^{-1}$ o nível a partir do qual ocorreu platô. Não foi verificada influência $(P>0,05)$ dos niveis de Ld sobre o consumo de ração diário (CRD) e ET. Os níveis de 12,0 e 12,5g $\mathrm{kg}^{-1}$ de Ld na dieta, correspondentes, respectivamente, ao consumo de lisina digestivel diário (CLdD), de 23,6 e 24,6g, proporcionam os melhores resultados de desempenho e área de olho de lombo de leitoas em fase de crescimento (63 aos 103 dias de idade).

Palavras-chave: carcaça, exigência nutricional, desempenho, suínos.

\section{ABSTRACT}

Eighty gilts $(24.2 \pm 1.52 \mathrm{~kg})$ with high genetic potential for lean gain were used in a randomized complete block design to evaluate five digestible lysine levels $(9,10,11,12$ and $13 \mathrm{~g} \mathrm{~kg}^{-1}$ ) during the growing phase (63 to 103 days of age). Gilts were housed in pair and fed their respective diets ad libitum. At the begging and the ending of the experimental period, gilts were weighed and scanned by ultrasound to measure loin area, as well as fat depth. The digestible lysine levels linearly increase $(P<0.05)$ average daily gain and loin area of gilts in growing phase. However, there was no difference for average daily gain at levels above $12 \mathrm{~g} \mathrm{~kg}^{-1}$ while the "Linear Response Plateau" model was that better fitted to the loin area data that stabilized in a plateau starting from the level of $12.5 \mathrm{~g} \mathrm{~kg}^{-1}$. The feed:gain ratio was improved $(P<0.01)$ quadratically until the estimated level of $11.9 \mathrm{~g} \mathrm{~kg}^{-1}$ of digestible lysine. There was no effects $(P>0.05)$ of the treatments on the fat depth and feed intake. The digestible lysine levels of 12 and $12.5 \mathrm{~g} \mathrm{~kg}^{-1}$, corresponding to the intake of $23.6 \mathrm{e}$ $24.6 \mathrm{~g}$ dia $^{-1}$, provide the best results of performance and loin area of growing gilts (63 to 103 days old).

Key words: carcass, performance, nutritional requirements, swine.

\section{INTRODUÇÃO}

Devido ao alto custo das fontes proteicas utilizadas em dietas para suínos, o conhecimento e/ ou atualização das exigências de aminoácidos para esses animais é fundamental para otimizar o uso dessas fontes. Um grande número de experimentos vem sendo conduzido na tentativa de quantificar os efeitos de diferentes níveis de Ld na dieta sobre o desempenho e características de carcaça dos suínos. No entanto, os resultados desses estudos não podem

'Programa de Pós-graduação em Zootecnia, Universidade Federal de Viçosa (UFV), 36570-000, Viçosa, MG, Brasil. E-mail: gcr90@yahoo.com.br. *Autor para correspondência.

"Departamento de Zootecnia, UFV, Viçosa, MG, Brasil.

"IIEmpresa de Pesquisa Agropecuária de Minas Gerais (EPAMIG), Viçosa, MG, Brasil.

${ }^{\mathrm{IV}}$ Faculdade de Medicina Veterinária e Zootecnia, Universidade Federal de Mato Grosso do Sul (UFMS), Campo Grande, MS, Brasil.

${ }^{\mathrm{v}}$ Universidade de Illinois Urbana Champaign (UIUC), Urbana, IL, EUA. 
ser generalizados, devido às variações nas condições experimentais, tais como genótipo, peso corporal e o sexo, que influenciam na eficiência de deposição de proteína e, consequentemente, na exigência de lisina (FRIESEN et al., 1994).

A busca por carcaças de melhor qualidade tem levado à seleção de suínos com alto potencial genético para desempenho e crescimento de tecido muscular. Esses animais, no entanto, apresentam maior exigência de aminoácidos, principalmente de lisina. Sendo assim, os níveis de Ld preconizados pela literatura podem estar desatualizados por não atender às exigências de aminoácidos essenciais dos atuais genótipos de suínos (GRANDHI \& NYACHOTI, 2002), evidenciando que a formulação das dietas devem ser periodicamente reavaliadas.

Além disso, tem sido verificado que as exigências dos suínos para o desempenho, medidas qualitativas e quantitativas de carcaça podem variar em função do sexo (ROSTAGNO et al., 2011). Leitoas possuem maior exigência de lisina e eficiência na deposição de proteína em relação aos machos castrados, no entanto, inferior aos machos inteiros. Tais diferenças são resultantes de mudanças endócrinas ocorridas durante todo o desenvolvimento sexual (BELLAVER \& VIOLA, 1997), sendo mais marcantes em suínos com peso superior a $25 \mathrm{~kg}$ ou acima de 63 dias de idade (QUINIOU et al., 2010).

Portanto, torna-se importante reavaliar as exigências nutricionais, principalmente de lisina, para animais de cada sexo e de acordo com o potencial genético para deposição de carne na carcaça. Dessa forma, este estudo teve como objetivo determinar a exigência de Ld para leitoas com alto potencial genético para deposição de carne na carcaça, em fase de crescimento (63 aos 103 dias de idade).

\section{MATERIAL E MÉTODOS}

Foram utilizadas 80 leitoas, híbridas comerciais (AGPIC $425 \times$ Camborough 25) com alto potencial genético para deposição de carne na carcaça, com peso inicial de $24,2 \pm 1,52 \mathrm{~kg}$ e 63 dias de idade, distribuídas em delineamento experimental de blocos ao acaso, composto de cinco tratamentos (níveis de Ld), oito repetições e dois animais por baia, o que foi considerada a unidade experimental. $\mathrm{Na}$ formação dos blocos, levou-se em consideração a localização dos animais no galpão.

Os tratamentos foram constituídos por uma dieta basal isenta de aminoácidos industriais e outras quatro dietas obtidas pela suplementação da dieta basal com L-Lisina $\mathrm{HCl} 78 \%$ e, quando necessário, com DL-Metionina 99\%, L-Treonina 98\%, L-Triptofano
98\% e L-Valina $96.5 \%$ em substituição ao amido, resultando em dietas com níveis de 9, 10,11, 12 e $13 \mathrm{~g}$ $\mathrm{kg}^{-1}$ de $\mathrm{Ld}$. As dietas experimentais foram formuladas a base de milho e farelo de soja, suplementadas com minerais, vitaminas e aminoácidos (Tabela 1) para atender às exigências nutricionais de suínos fêmeas de alto potencial genético com desempenho superior em fase de crescimento (30 a 60kg), exceto para a Ld, mantendo-se as relações aminoacídicas entre a lisina e os demais aminoácidos essenciais, de acordo com o padrão de proteína ideal proposto por ROSTAGNO et al. (2005).

Os animais foram alojados em galpão de alvenaria com piso de concreto, coberto com telhas de amianto. As baias continham comedouros semiautomáticos, bebedouros tipo chupeta e dispunham de área de $1,87 \mathrm{~m}^{2}$ por animal. As condições ambientais no interior do galpão experimental foram monitoradas e registradas três vezes ao dia $(7,12$ e $17 \mathrm{~h})$ por meio de termômetro de globo negro e a cada hora por meio de uma estação meteorológica (data logger ${ }^{\circledR}$, modelo 3030.15, 433MHz, TFA, Alemanha), instrumentos estes mantidos em uma baia vazia no centro do galpão. Os valores registrados foram convertidos no índice de temperatura de globo e umidade (ITGU) para a caracterização do ambiente.

As rações e a água foram fornecidas à vontade aos animais durante o período experimental. Para determinação do CRD, CLdD, GPD e CA, foram pesadas periodicamente as rações, sobras e desperdícios e os animais individualmente, no início e ao final do período experimental.

Para avaliação da carcaça, foi realizada, no início e ao final do período experimental, após a pesagem dos animais, a avaliação da AOL e ET, através de aparelho de ultrassom (Aloka SSD 500). As imagens foram coletadas entre a décima e a décima primeira costela, conforme preconizado pelo National Swine Improvement Federation Guidelines. A partir das imagens obtidas, a AOL e a ET foram calculadas, utilizando-se o programa computacional Biosoft toolbox for Swine (Biotronics Inc.).

Para verificar o ajuste das equações obtidas, considerou-se a menor soma dos quadrados dos desvios, a significância do teste $\mathrm{F}$ sob a hipótese H0: $\beta 0=\beta 1=\beta 2=0$ e os coeficientes de determinação ( $\mathrm{R} 2=\mathrm{SQ}$ regressão/SQ tratamentos). Os dados foram analisados por meio dos procedimentos GLM e NLIN do SAS (Statistical Analysis System Institute, Inc., Cary, NC, USA). Para análise de variância (ANOVA), o procedimento GLM foi usado para testar os efeitos linear e quadrático dos níveis de Ld nas variáveis resposta, através do método de polinômios 
Tabela 1 - Composições centesimais e nutricionais calculadas das dietas experimentais para leitoas em fase de crescimento.

\begin{tabular}{|c|c|c|c|c|c|}
\hline \multirow{2}{*}{ Ingredientes } & \multicolumn{5}{|c|}{ - } \\
\hline & 9 & 10 & 11 & 12 & 13 \\
\hline Milho grão moído & 63,989 & 63,989 & 63,989 & 63,989 & 63,989 \\
\hline Farelo de soja $45 \%$ & 30,745 & 30,745 & 30,745 & 30,745 & 30,745 \\
\hline Óleo de soja & 1,425 & 1,425 & 1,425 & 1,425 & 1,425 \\
\hline Fosfato bicálcico & 1,207 & 1,207 & 1,207 & 1,207 & 1,207 \\
\hline Amido & 1,063 & 0,889 & 0,631 & 0,350 & 0,000 \\
\hline Calcário calcítico & 0,631 & 0,631 & 0,631 & 0,631 & 0,631 \\
\hline Sal comum & 0,405 & 0,405 & 0,405 & 0,405 & 0,405 \\
\hline Premix vitamínico/mineral ${ }^{1}$ & 0,400 & 0,400 & 0,400 & 0,400 & 0,400 \\
\hline L-Lisina $\mathrm{HCl} 78 \%$ & 0,000 & 0,129 & 0,258 & 0,387 & 0,516 \\
\hline DL-Metionina $99 \%$ & 0,000 & 0,039 & 0,099 & 0,160 & 0,221 \\
\hline L-Treonina $98 \%$ & 0,000 & 0,006 & 0,075 & 0,144 & 0,212 \\
\hline L-Triptofano $98 \%$ & 0,000 & 0,000 & 0,000 & 0,008 & 0,027 \\
\hline L-Valina $96,5 \%$ & 0,000 & 0,000 & 0,000 & 0,014 & 0,087 \\
\hline Sulfato de colistina $8 \%$ & 0,125 & 0,125 & 0,125 & 0,125 & 0,125 \\
\hline Butilhidroxitolueno & 0,010 & 0,010 & 0,010 & 0,010 & 0,010 \\
\hline \multicolumn{6}{|c|}{--Composição nutricional calculada ${ }^{2}$} \\
\hline Energia metabolizável $\left(\mathrm{Kcal} \mathrm{kg}^{-1}\right)$ & 3.230 & 3.230 & 3.230 & 3.230 & 3.230 \\
\hline Proteína bruta $(\%)$ & 19,24 & 19,24 & 19,24 & 19,24 & 19,24 \\
\hline Lisina digestivel $(\%)$ & 0,900 & 1,000 & 1,100 & 1,200 & 1,300 \\
\hline Met. + Cis. digestivel (\%) & 0,562 & 0,600 & 0,660 & 0,720 & 0,780 \\
\hline Treonina digestivel $(\%)$ & 0,644 & 0,650 & 0,715 & 0,780 & 0,845 \\
\hline Triptofano digestivel (\%) & 0,208 & 0,208 & 0,208 & 0,216 & 0,234 \\
\hline Valina digestivel $(\%)$ & 0,815 & 0,815 & 0,815 & 0,828 & 0,897 \\
\hline Isoleucina digestível (\%) & 0,739 & 0,739 & 0,739 & 0,739 & 0,739 \\
\hline Cálcio $(\%)$ & 0,631 & 0,631 & 0,631 & 0,631 & 0,631 \\
\hline Fósforo disponivel (\%) & 0,330 & 0,330 & 0,330 & 0,330 & 0,330 \\
\hline Sódio $(\%)$ & 0,180 & 0,180 & 0,180 & 0,180 & 0,180 \\
\hline
\end{tabular}

${ }^{1}$ Conteúdo mínimo por kg de produto: 2.000.000UI de Vitamina A; 300.000UI de Vitamina D3; 5.000UI de Vitamina E; 625mg de Vitamina K3; $5.000 \mathrm{mcg}$ de Vitamina B12; $1.000 \mathrm{mg}$ de Vitamina B2; $12,5 \mathrm{mg}$ de Biotina; $2.500 \mathrm{mg}$ de Ácido pantotênico; $6.250 \mathrm{mg}$ de Niacina; $500 \mathrm{mg}$ de Butilhidroxitolueno; $250 \mathrm{mg}$ de Vitamina B1; $500 \mathrm{mg}$ de Vitamina B6;150mg de Ácido fólico; $60 \mathrm{~g}$ de Colina; 12,5g de Vitamina C; $125 \mathrm{mg}$ de Cobalto; $125 \mathrm{mg}$ de Selênio; $17,5 \mathrm{~g}$ de Ferro; $5.000 \mathrm{mg}$ de Cobre; $10 \mathrm{~g}$ de Manganês; $20 \mathrm{~g}$ de Zinco; $200 \mathrm{mg}$ de Iodo e Veículo q.s.p. 1000g. ${ }^{2}$ Composição nutricional calculada com base em ROSTAGNO ct al. (2005).

ortogonais, em um modelo contendo os efeitos fixos de peso inicial (covariável), níveis de Ld e lado do galpão (duas localizações). Para a variável AOL, o procedimento NLIN foi usado para ajustar um modelo "Linear Response Plateau", contendo os níveis de Ld (ROBBINS et al., 2006). Efeitos foram considerados significativos ao nível de 5\% de significância. Para os dados de desempenho, a unidade experimental foi representada pela baia e, para os dados de ultrassom, pelo suíno.

\section{RESULTADOS E DISCUSSÃO}

Durante o período experimental, a temperatura do ar registrada no interior do galpão foi de $24,9 \pm 3,9^{\circ} \mathrm{C}$, a umidade relativa foi de $80,6 \pm 11,8 \%$ e a temperatura de globo negro foi de $25,5 \pm 3,6^{\circ} \mathrm{C}$, correspondendo a um ITGU calculado de 74,5 $\pm 4,2$. Considerando que, segundo SAMPAIO et al. (2004), a temperatura crítica máxima para essa categoria animal é de $27^{\circ} \mathrm{C}$ e que MANNO et al. (2006) caracterizou o ambiente de 72,3 como de termoneutralidade para suínos de 30 aos $60 \mathrm{~kg}$, pode-se inferir que os animais foram submetidos a ambiente de conforto térmico no presente estudo.

Os níveis de lisina digestível não influenciaram $(\mathrm{P}>0,05)$ no $\mathrm{CRD}$ dos animais (Tabela 2). Esse resultado está coerente com os obtidos por FONTES et al. (2000) e ABREU et al. (2007), que também não constataram efeito dos níveis de

Ciência Rural, v.43, n.5, mai, 2013. 
Ld (9-12 e 8-11 $\mathrm{g} \mathrm{kg}^{-1}$, respectivamente) sobre a ingestão voluntária de alimento de suínos em fase de crescimento. Por outro lado, MAIN et al. (2008), testando níveis de 8,0 a $13,5 \mathrm{~g} \mathrm{~kg}^{-1}$ de $\mathrm{Ld}$, encontraram efeito linear negativo dos tratamentos sobre o CRD de leitoas dos 35 aos $60 \mathrm{~kg}$.

A divergência de resultados observada entre os trabalhos pode estar relacionada a diferenças na composição da dieta. Enquanto nos estudos em que não foi observada variação significativa no CRD dos animais, os níveis de $\mathrm{Ld}$ foram obtidos com a inclusão de aminoácido industrial, no de MAIN et al. (2008), os níveis de Ld foram alcançados aumentando a quantidade de farelo de soja na dieta. Fundamentando essa hipótese, HEO et al. (2012) relataram que altos níveis de farelo de soja podem influenciar negativamente na ingestão voluntária de alimentos pelos leitões.

O CLdD aumentou $(\mathrm{P}<0,05)$ de forma linear à medida que se elevou o seu nível na dieta, conforme a equação: $\hat{Y}=-0,747+2,028 \operatorname{Ld}\left(r^{2}=0,98\right)$, estando de acordo com resultados reportados na literatura (FONTES et al., 2000; ABREU et al., 2007; ZANGERONIMO et al., 2009). O aumento da ingestão de lisina diário justifica-se em razão da concentração deste na dieta, uma vez que não se verificou variação significativa no consumo voluntário de ração dos animais.

O GPD das leitoas aumentou $(\mathrm{P}<0,05)$ de forma linear, de acordo com os níveis de inclusão de Ld nas dietas, conforme a equação: $\hat{Y}=791,546+16,703$ Ld $\left(r^{2}=0,88\right)$. Apesar dessa variação, constata-se que não houve aumento no valor absoluto do GPD a partir do nível de $12 \mathrm{~g}$ de $\mathrm{Ld}$, correspondente a um CLdD de 23,6g, indicando ser este o nível para melhor resposta de ganho de peso. Os níveis de Ld influenciaram $(\mathrm{P}<0,01)$ na $\mathrm{CA}$, que melhorou de forma quadrática até o nível estimado de $11,9 \mathrm{~g} \mathrm{~kg}^{-1}$ de $\mathrm{Ld}$, correspondente ao consumo de $23,4 \mathrm{~g} \mathrm{dia}^{-1}$ de Ld (Figura 1a).

Resultados positivos dos níveis de $\mathrm{Ld}$ dietéticos sobre o GPD e CA de leitoas também foram relatados por MAIN et al. (2008), que obtiveram as melhores respostas de desempenho no nível $10,2 \mathrm{~g}$ $\mathrm{kg}^{-1}$ de Ld correspondente ao CLdD de 19,8g dia-1. Por sua vez, FONTES et al. (2000) e ABREU et al. (2007) observaram melhora da CA até o nível de 13 e $11 \mathrm{~g} \mathrm{~kg}^{-1}$ de Ld, respectivamente, correspondendo a um consumo de 22,1 e 21,9g dia ${ }^{-1}$ de Ld, entretanto, sem alteração significativa no GPD dos animais.

Apesar da similaridade de resultados entre os trabalhos, constatou-se que a exigência de Ld em $\mathrm{g} \mathrm{dia}^{-1}$ das leitoas para expressarem a melhor resposta de CA e GPD, neste estudo, ficou acima da obtida pelos demais autores. Essa diferença de resposta de desempenho dos animais em razão da variação do nível de Ld na dieta pode estar relacionada, entre outros fatores, ao potencial genético dos animais quanto à capacidade de deposição de carne na carcaça. Segundo FRIESEN et al. (1994), suínos selecionados para maior deposição proteica apresentam maior capacidade de crescimento e eficiência alimentar, o que resulta em maior exigência de lisina.

Além do genótipo, outros fatores como padrão sanitário e ambiente térmico podem alterar a exigência de lisina dos suínos. WILLIAMS et al. (1997) evidenciaram que a ativação permanente ou temporária do sistema imunológico dos suínos interfere na sua exigência nutricional de lisina. Em adição, KERR et al. (2003) constataram que a ativação do sistema termorregulatório de suínos pode influenciar na sua exigência em aminoácidos.

Tabela 2 - Desempenho e características de carcaça de leitoas, em fase de crescimento, em função dos níveis de lisina digestivel na dieta.

\begin{tabular}{|c|c|c|c|c|c|c|}
\hline \multirow{2}{*}{ Variáveis } & \multirow[b]{2}{*}{9} & \multirow[b]{2}{*}{10} & \multirow[b]{2}{*}{11} & \multirow[b]{2}{*}{12} & \multirow[b]{2}{*}{13} & \multirow{2}{*}{$\mathrm{CV}(\%)$} \\
\hline & & & & & & \\
\hline Peso inicial (kg) & 24,2 & 24,2 & 24,2 & 24,2 & 24,3 & 2,58 \\
\hline Peso final $(\mathrm{kg})^{1}$ & 62,1 & 62,5 & 62,8 & 64,5 & 64,3 & 3,23 \\
\hline Consumo de ração diário $(\mathrm{g})$ & 2.004 & 1.911 & 1.929 & 1.964 & 1.994 & 6,30 \\
\hline Consumo de $\mathrm{Ld}\left(\mathrm{g} \mathrm{dia}^{-1}\right)^{1}$ & 18,0 & 19,1 & 21,2 & 23,6 & 25,9 & 6,11 \\
\hline Ganho de peso diário $(\mathrm{g})^{1}$ & 946 & 956 & 965 & 1007 & 1002 & 5,34 \\
\hline Conversão alimentar ${ }^{2}$ & 2,12 & 2,00 & 2,01 & 1,96 & 1,99 & 4,00 \\
\hline Área de olho de lombo $\left(\mathrm{cm}^{2}\right)^{1}$ & 39,4 & 40,2 & 40,5 & 42,5 & 42,6 & 6,64 \\
\hline Espessura de toucinho $(\mathrm{mm})$ & 13,5 & 13,0 & 13,5 & 13,2 & 13,8 & 10,9 \\
\hline
\end{tabular}

${ }^{1}$ Efeito Linear $(\mathrm{P}<0,05) .{ }^{2}$ Efeito Quadrático $(\mathrm{P}<0,01)$. 


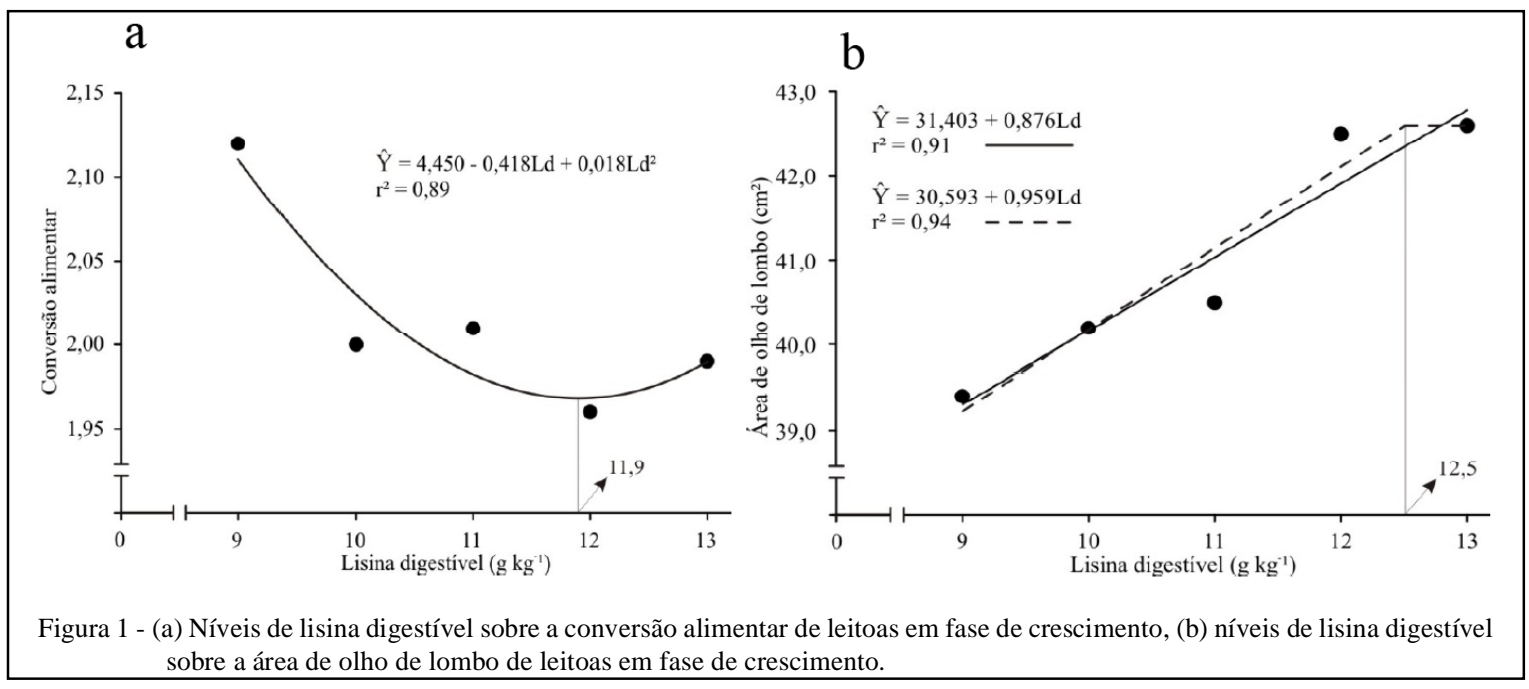

A melhora verificada na CA dos animais, no presente estudo, seria um indicativo de alteração na composição do ganho de peso dos animais com aumento proporcional na deposição de proteína.

O nível de $12,0 \mathrm{~g} \mathrm{~kg}^{-1}$ de $\mathrm{Ld}$ na dieta, correspondente ao consumo $23,6 \mathrm{~g} \mathrm{dia}^{-1}$ de $\mathrm{Ld}$, que proporcionou melhor resposta de desempenho (GPD e CA) das leitoas neste estudo, ficou acima da recomendação de $20,0 \mathrm{~g}$ dia $^{-1}$ preconizada por ROSTAGNO et al. (2011), sendo um indicativo de que o nível atualmente recomendado pode não atender às exigências para maximizar o desempenho das leitoas na fase de crescimento.

Não houve diferença $(\mathrm{P}>0,05)$ para $\mathrm{AOL}$ $\left(17,3 \pm 0,57 \mathrm{~cm}^{2}\right)$ e ET $(8,7 \pm 0,37 \mathrm{~mm})$ dos animais entre os tratamentos no início do período experimental. No entanto, ao final desse período (103 dias de idade), a AOL aumentou $(\mathrm{P}<0,05)$ de forma linear, à medida que se elevou a concentração de Ld na dieta. Como não ocorreu variação considerável para AOL dos animais entre os dois maiores níveis de Ld, o modelo Linear Response Plateau foi o que melhor se ajustou aos dados, estimando em $12,5 \mathrm{~g} \mathrm{~kg}^{-1}$ o nível ótimo de Ld, a partir do qual ocorreu platô (Figura 1b), correspondendo a um consumo estimado de 24,6g $\operatorname{dia}^{-1}$ de Ld.

De forma similar, BIKKER et al. (1994) e DE LA LLATA et al. (2007), trabalhando com leitoas, constataram melhora significativa da profundidade de lombo e deposição de proteína na carcaça, com aumento de Ld nas dietas. No entanto, MAIN et al. (2008) não verificaram efeito dos níveis de $\mathrm{Ld}$ da dieta sobre a AOL em leitoas na fase de crescimento. A inconsistência de resultados observada entre os trabalhos revela a diferença na genética dos animais utilizados nos estudos quanto ao potencial de deposição de carne na carcaça, o que justifica as variações de exigência de Ld dos suínos nos diferentes genótipos.

No presente estudo, o nível de Ld que proporcionou melhor resultado de desempenho $(12,0 \mathrm{~g}$ $\mathrm{kg}^{-1}$ ) está abaixo do obtido para maior deposição de carne $\left(12,5 \mathrm{~g} \mathrm{~kg}^{-1}\right)$. Em trabalho conduzido para determinar a exigência de $\mathrm{Ld}$ de leitoas em fase de crescimento, BIKKER et al. (1994) e DE LA LLATA et al. (2007) também observaram maior exigência de Ld para deposição de proteína na carcaça, quando comparado à eficiência de utilização do alimento para GPD.

Os níveis de Ld não influenciaram $(\mathrm{P}>0,05)$ nos valores de ET. Segundo CISNEROS et al. (1996), a mensuração da AOL e da ET com o ultrassom fornece medidas acuradas da quantidade de músculo e gordura na carcaça. Sendo assim, a não alteração da ET das leitoas, associada ao aumento linear da AOL, em resposta aos níveis de Ld na dieta, é indicativo de melhora na relação proteína/gordura na carcaça.

Tem sido relatado que as deposições de proteína e de gordura são inversamente relacionadas (FÁVERO \& BELLAVER, 2001). No entanto, isso não se confirmou nesse estudo, uma vez que o aumento observado na AOL não resultou em redução da ET. Esses resultados corroboram os de ABREU et al. (2007) e ZANGERONIMO et al. (2009), que também obtiveram resposta nas variáveis ligadas à deposição proteica sem a correspondente variação na deposição de gordura. $\mathrm{O}$ padrão de resposta verificado para a AOL e ET, no presente estudo, está consistente com a melhora ocorrida na CA, confirmando a modificação na composição do ganho de peso dos animais, com melhora na relação carne:gordura na carcaça. 


\section{CONCLUSÃO}

Leitoas com alto potencial genético para deposição de carne na carcaça, em fase de crescimento (63 aos 103 dias de idade), exigem os níveis de 12,0 e $12,5 \mathrm{~g} \mathrm{~kg}^{-1}$ de lisina digestível na dieta, correspondentes, respectivamente, ao consumo de 23,6 e 24,6 dia $^{-1}$ de lisina digestível, para melhores resultados de desempenho e área de olho de lombo.

\section{AGRADECIMENTO}

À Fundação de Amparo à Pesquisa do Estado de Minas Gerais (FAPEMIG), pelo financiamento do projeto de pesquisa.

\section{COMITÊ DE ÉTICA}

Aprovado pela comissão de ética no uso de animais/ UFMS, sob protocolo n.427/2012.

\section{REFERÊNCIAS}

ABREU, M.L.T. et al. Níveis de lisina digestível em rações, utilizando-se o conceito da proteína ideal, para suínos machos castrados de alto potencial genético para deposição de carne magra na carcaça, dos 30 a $60 \mathrm{~kg}$. Revista Brasileira de Zootecnia, v.36, p.62-67, 2007. Disponível em: <http://www.scielo.br/ scielo.php?pid $=$ S1516-35982007000100008\&script $=$ sci_arttext $>$. Acessoem: 21 abr. 2012. doi: 10.1590/S1516-35982007000100008.

BELLAVER, C.; VIOLA, E.S. Qualidade de carcaça, nutrição e manejo nutricional. In: CONGRESSO BRASILEIRO DE VETERINÁRIOS ESPECIALISTAS EM SUÍNOS, 8., 1997, Foz do Iguaçu, PR. Anais... Foz do Iguaçu: ABRAVES, 1997. p.152-158.

BIKKER, P.M.W. et. al. Digestible lysine requirement of gilts with high genetic potential for lean gain, in relation to the level of energy intake. Journal of Animal Science, v.72, p.17441753, 1994. Disponível em: <http://www.ncbi.nlm.nih.gov/ pubmed/7928754>. Acesso em: 10 jun. 2012.

CISNEROS, F. et al. Comparison of transverse and longitudinal real-time ultrasound scans for prediction of lean cut yields and fatfree lean content in live pigs. Journal of Animal Science, v.74, p.2566-2576, 1996. Disponível em: <http://www.ncbi.nlm.nih. gov/pubmed/8923171>. Acesso em: 13 jul. 2012.

DE LA LLATA, M. et al. Effects of increasing lysine to calorie ratio and added fat for growing-finishing pigs reared in a commercial environment: I. Growth performance and carcass characteristics. Professional Animal Scientist, v.23, p.417-428, 2007. Disponível em: <http://pas.fass.org/content/23/4/417.abstract>. Acesso em: 10 jul. 2012.

FÁVERO, J.A.; BELLAVER, C. Produção de carne de suínos. In: CONGRESSO BRASILEIRO DE CIÊNCIA E TECNOLOGIA DE CARNES, 2001, Campinas, SP. Anais... Campinas: CTC/ITAL, 2001. p.2-25. Disponível em: <www.cnpsa.embrapa.br/sgc/sgc publicacoes/publicacao_f0q38d0g.pdf >. Acesso em: 10 jul. 2012.

FONTES, D.O. et al. Níveis de lisina para leitoas selecionadas geneticamente para deposição de carne magra, dos 30 aos $60 \mathrm{~kg}$, mantendo constante a relação entre lisina e metionina+cistina, treonina, triptofano, isoleucina e valina. Revista Brasileira de Zootecnia, v.29, p.776-783, 2000. Disponível em: <http://www. revista.sbz.org.br/artigo/index.php?artigo $=2548>$. Acesso em: 12 mar. 2011. doi: 10.1590/S1516-35982000000300020.

FRIESEN, K.G. et al. Effects of the interrelationship between genotype, sex, and dietary lysine on growth performance and carcass composition in finishing pigs fed to either 104 or 127 kilograms. Journal of Animal Science, v.72, p.946-954, 1994. Disponível em: <http://www.ncbi.nlm.nih.gov/pubmed/8014161>. Acesso em: 12 mar. 2011.

GRANDHI, R.R.; NYACHOTI, C.M. Effect of true ileal digestible dietary methionine to lysine ratios on growth performance and carcass merit of boars, gilts and barrows selected for low backfat. Canadian Journal of Animal Science, v.82, p.399-407, 2002. Disponível em: <http://pubs.aic.ca/doi/pdf/10.4141/A01-015>. Acesso em: 19 ago. 2011

HEO, J.M. et al. Gastrointestinal health and function in weaned pigs: a review of feeding strategies to control post-weaning diarrhoea without using in-feed antimicrobial compounds. Journal of Animal Physiology and Animal Nutrition, p.1-31, 2012. Disponível em: <http://onlinelibrary.wiley.com/doi/10.1111/ j.1439-0396.2012.01284.x/pdf>. Acesso em: 29 mar. 2012. doi: 10.1111/j.1439-0396.2012.01284.

KERR, B.J. et al. Influences of dietary protein level, amino acid supplementation and environmental temperature on performance, body composition, organ weights and total heat production of growing pigs. Journal of Animal Science, v.81, p.19982007, 2003. Disponível em: <http://www.ncbi.nlm.nih.gov/ pubmed/12926782>. Acesso em: 19 ago. 2011.

MAIN, R.G. et al. Determining an optimum lysine:calorie ratio for barrows and gilts in a commercial finishing facility. Journal of Animal Science, v.86, p.2190-2207, 2008. Disponível em: <http:// www.ncbi.nlm.nih.gov/pubmed/18469046>. Acesso em: 29 mar. 2012. doi: 10.2527/jas.2007-0408.

MANNO, M.C. et al. Efeitos da temperatura ambiente sobre o desempenho de suínos dos 30 aos $60 \mathrm{~kg}$. Revista Brasileira de Zootecnia, v.35, p.471-477, 2006. Disponível em: <http://www. ncbi.nlm.nih.gov/pubmed/18469046>. Acesso em: 12 mar. 2011. doi: $10.2527 /$ jas.2007-0408.

QUINIOU, N. et al. Impact of the non castration of male pigs on growth performance and behavior-comparison with barrows and gilts. In: ANNUAL MEETING OF THE EUROPEAN ASSOCIATION FOR ANIMAL PRODUCTION, 61., 2010, Herakilion. Proceedings... Creye Island, Greece: Herakilion, 2010. p.136. Disponível em: <http://www.eaap.org/Previous_ Annual_Meetings/2010Crete/Papers/17_Quiniou.pdf >. Acesso em: 12 mar. 2011

ROBBINS, K.R. et al. Estimation of nutrient requirements using broken-line regression analysis. Journal of Animal Science, v.84, p.155-165, 2006. Disponível em: <http://www.ncbi.nlm.nih.gov/ pubmed/16582088>. Acesso em: 21 abr. 2012.

ROSTAGNO, H.S. et al. Tabelas brasileiras para aves e suínos; composição de alimentos e exigências nutricionais. 2.ed. Viçosa: UFV, Imprensa Universitária, 2005. 186p. 
ROSTAGNO, H.S. et al. Tabelas brasileiras para aves e suínos; composição de alimentos e exigências nutricionais. 3.ed. Viçosa: UFV, Imprensa Universitária, 2011. 252p.

SAMPAIO, C.A.P. et al. Avaliação do ambiente térmico em instalações para crescimento e terminação de suínos utilizando os índices de conforto térmico nas condições tropicais. Ciência Rural, v.34, p.785-790, 2004. Disponível em: <http://www.scielo. $\mathrm{br} / \mathrm{scielo}$.php? pid=S0103-84782004000300020\& script $=\mathrm{sci}_{-}$ arttext $>$. Acesso em: 21 fev. 2012. doi: 10.1590/S010384782004000300020 .
WILLIAMS, N.H. et al. Effect of chronic immune system activation on the rate, efficiency, and composition of growth and lysine needs of pigs fed from 6 to $27 \mathrm{~kg}$. Journal of Animal Science, v.75, p.2463-2471, 1997. Disponível em: <http://www. ncbi.nlm.nih.gov/pubmed/9303465>. Acesso em: 10 fev. 2012.

ZANGERONIMO, M.G. et al. Desempenho e características de carcaça de suínos dos 20 aos $50 \mathrm{~kg}$ recebendo rações com reduzido teor de proteína bruta e diferentes níveis de lisina digestível verdadeira. Ciência Rural, v.39, p. 1507-1513, 2009. Disponível em: <http:// www.scielo.br/pdf/cr/2009nahead/a175cr444.pdf>. Acesso em: 12 jun. 2012. 